\section{ABDOMINAL PAIN: DO WE MAKE APPROPRIATE REFERRALS TO THE NORTHERN IRELAND PAEDIATRIC SURGICAL SERVICE?}

\section{J.-A. Maney ${ }^{1}$, W. McCallion²}

${ }^{1}$ Emergency Department, ${ }^{2}$ Consultant Paediatric Surgery, Royal Belfast Hospital for Sick Children, Belfast, UK

Background \& Aims: Abdominal pain in children is a common presentation to the emergency department and a frequent cause of referral to the paediatric surgical service. The aim of the study is to analyse current practice and referral patterns to the surgical service.

Method: Retrospective analysis of records of children presenting to the Royal Belfast Hospital for Sick Children with abdominal pain. We identified children presenting to the emergency department and referred to the surgical service for a period of 2 weeks in November 2009.

Results: There were 57 cases identified. The differential diagnoses included $3 \%$ of patients with acute appendicitis, confirmed at histology.

There were 11 surgical referrals, six from the emergency department and five transfers from district general hospitals. The transfers were all discharged; none of these patients had been reviewed or discussed with a senior clinician prior to transfer.

Clinical assessment and management in general was good and well documented.

Conclusion: Abdominal pain is a common presentation and if a surgical diagnosis is missed can have devastating consequences.

RBHSC offers a tertiary referral service for all paediatric surgery in Northern Ireland.

From this study we have shown that transfers from district general hospitals were inappropriate as all cases were discharged from the emergency department following assessment by the surgeon. Referrals to the tertiary surgical service should be reviewed and discussed with a senior clinician prior to transfer. Referrals made from the emergency department were appropriate and were all reviewed by a senior clinician prior to referral.

\section{RESOURCE IMPLICATIONS IN THE MANAGEMENT OF PENETRATING TRAUMA IN CHILDREN}

\author{
A. Cho ${ }^{1}$, A. Cambell ${ }^{1}$, S. Phelps ${ }^{1}$, S. Rajan ${ }^{2}$,
} N. Edmonds ${ }^{2}$

${ }^{1}$ Paediatric Surgery, ${ }^{2}$ Paediatric Critical Care Unit, Barts and the London Children's Hospital, London, UK

Aims: To identify the resource implications in the management of penetrating trauma in children (aged $<16$ years of age)

Methods: The paediatric trauma database of all children was used to identify all children with penetrating trauma presenting to our A\&E, a major urban trauma centre in London. The study period was over 19 months till December 2009.

Results: During this period, 252 children presented to the Emergency Department following major trauma call activation. 32 of these patients admitted with penetrating trauma. Case-notes were available for 23 patients (71\%) The average age was 14.3 years. $91 \%$ were male. 2 patients had gun shot wounds. $78 \%$ presented between 18:00 and 08:00.

A total of 70 penetrating wounds were documented. The commonest sites were posterior chest $(29 \%)$, upper limb (20\%) \& lower limb (17\%). 8 had chest drains inserted in the emergency department.

Radiological investigations: CXR (96\%), FAST scan $(57 \%)$ \& CT scan $(70 \%) .13$ patients $(57 \%)$ underwent an emergency operation under general anaesthetic: Exploration \& wound debridement (6), tendon repair (2), thoractomy (1) \& laparotomy (4). Cross specialties involved included PICU (26\%), orthopaedics (23\%), adult trauma surgeons (13\%), plastic surgery (9\%), CAHMS / social services $(74 \%)$. Average length of stay in the PICU was 2.5 days and 3.1 hospital days. There were three deaths of the 32 patients

Conclusions: Penetrating trauma occurring in the paediatric population is of increasing prevalence. This study highlights the huge resources required to manage such patients. 